\title{
Effects of Different Levels of Fatigue on Vertical Jump Performance, Vertical Stiffness, and Intralimb Coordination
}

\author{
Débora Aparecida Knihs'1, Jonathan Ache Dias², Juliano Dal Pupo
}

Affiliations: ${ }^{1}$ Federal University of Santa Catarina, Sports Center, Physical Education Department, Florianopolis, Brazil, ${ }^{2}$ Catarinense Federal Institute, Research Group on Technology, Sports and Rehabilitation, Araquari, Brazil

Correspondence: D. A. Knihs, Federal University of Santa Catarina, Sports Center, Deputado Antônio Edu Vieira, Pantanal 88040-900, Florianopolis, Brazil. E-mail: deboraknihs@gmail.com

\begin{abstract}
This study aimed to investigate the acute effects of different levels of muscular fatigue on vertical jump performance, vertical stiffness, and intralimb coordination. Seventeen physically active men performed two fatigue protocols (low volume and high volume) composed of continuous vertical jumps on separate weeks. Jump height, vertical stiffness, and intralimb coordination were measured during countermovement vertical jumps prior to and immediately following the fatigue protocols. The jumps were performed on a force plate and filmed with high-speed cameras. The continuous relative phase was calculated as a measure of intralimb coordination. Mixed-model ANOVA was used to compare the variables between conditions and times. The fatigue index was greater in the high-volume protocol $(27 \pm 12 \%)$ than in the low-volume protocol (16 $\pm 7 \%)$. Jump height decreased $(p \leq 0.01)$ after the high-volume protocol. Vertical stiffness decreased $(p=0.05)$, and the continuous relative phase of thigh-shank coupling in the ascent phase of countermovement jumps increased $(p=0.04)$ after both protocols. In conclusion, jump performance was only affected by higher fatigue indexes, while vertical stiffness and intralimb coordination were affected similarly irrespective of the fatigue levels.
\end{abstract}

Keywords: stretch-shortening cycle, jump height, spring-mass model, continuous relative phase, motor control, biomechanics

$@$ MJSSMontenegro

FATIGUE EFFECTS ON PERFORMANCE, STIFFNESS, AND COORDINATION

http://mjssm.me/?sekcija=article\&artid=226

Cite this article: Knihs, D. A., Ache-Dias J., \& Dal Pupo J. (2022). Effects of Different Levels of Fatigue on Vertical Jump Performance, Vertical Stiffness, and Intralimb Coordination. Montenegrin Journal of Sports Science and Medicine, 11 (1), 9-14. https://doi.org/10.26773/mjssm.220301

\section{Introduction}

Repetitive, intense exercises involving the stretching-shortening cycle (SSC), such as vertical jumps, generate a high rate of mechanical work and consequently induce substantial muscular fatigue (Komi, 2000). It has been shown that this kind of exercise can lead to acute and long-term impairments of muscle function, which directly affect task perfor- mance (Byrne et al., 2004; Nicol et al., 2006). Generally, SSC performance impairment has been associated with changes in parameters related to the motor control of vertical jumps, such as lower limb stiffness and coordination of joints or body segments (Dal Pupo et al., 2013; Rodacki et al., 2002).

Many studies in the literature investigate the effects of fatigue on lower limb stiffness and coordination, but the re- 
sults are inconsistent. Some studies have shown that lower limb stiffness remains unchanged with fatigue (Kuitunen et al., 2007; Mudie et al., 2016; Padua et al., 2006), while decreased stiffness has also been reported in the literature (Dal Pupo et al., 2013; Horita et al., 1996; Lazaridis et al., 2018; Zhang et al., 2018). These alterations have been attributed to a reduction in the pre-activation and the stretching reflex of certain muscles responsible for stiffness regulation (Kuitunen et al., 2002; Lazaridis et al., 2018). In terms of performance, maintaining an optimal level of stiffness is required for the effective use of the elastic energy in the SSC function; however, stiffness reduction with fatigue observed in some studies has been suggested as a protective neural mechanism against injuries that may occur upon high ground impact (Hughes \& Watkins, 2008).

Similar results have been observed for intralimb coordination under SSC fatigue conditions. Changes in the coordination of the lower limbs due to fatigue have been observed as a compensation of the motor control system, aiming to maintain task performance (Dal Pupo et al., 2013; James et al., 2006; Lazaridis et al., 2018; Madigan \& Pidcoe, 2003) or as a protective mechanism against injuries (Hughes \& Watkins, 2008; Madigan \& Pidcoe, 2003). In contrast, other studies have shown that coordination does not change even under fatigue conditions due to the existence of a pre-programmed coordination pattern, which maintains the execution of movement through fixed neural commands (Rodacki et al., 2001).

It is known that muscular fatigue is task-dependent; thus, factors such as the type of muscle contraction and the workload training (volume and intensity) may directly influence the response to a bout of fatiguing exercise (Enoka \& Stuart, 1992; Kuitunen et al., 2007; Nicol et al., 2006). Generally, more intense or longer exercises (e.g., jump protocols of high volume) causes more fatigue and consequently induce greater impairment in muscle function when compared to less intense or lower volume exercises (Nicol et al., 2006), but this fatigue response seems to be more evident in neuromuscular performance (i.e., reduction in strength/power production capacity). In contrast, the fatigue effects on movement control parameters (e.g., stiffness or segmental coordination) have been suggested as non-uniform in different workloads (Lazaridis et al., 2018) and are not well understood in the literature. Identifying how movement control and performance parameters are affected when athletes are exposed to different workloads (e.g., during training or competition), for example, is particularly important in determining recovery time aiming to maximize performance and avoiding possible injury risks (McMahon et al., 2012; Rodacki et al., 2002).

Thus, the objective of this study was to investigate the acute effects of different muscular fatigue levels on vertical jump performance, vertical stiffness, and intralimb coordination. We hypothesize that there will be changes in jump performance and parameters related to the movement control (i.e., vertical stiffness and intralimb coordination) but depending on the fatigue levels.

\section{Methods}

Study design

This study has a crossover design in which participants performed two fatigue protocols using continuous vertical jumps (low-volume and high-volume protocols) on separate days. Dependent variables (jump height, vertical stiffness, and continuous relative phase) were measured in the countermovement jump prior to and immediately following the fatigue protocols. The participants performed both protocols, the low-volume followed by the high-volume protocol, separated by a one-week interval.

\section{Participants}

Seventeen healthy male adults (age: $26.8 \pm 3.3$ years, body mass: $79.3 \pm 11.5 \mathrm{~kg}$, height: $181.2 \pm 6.4 \mathrm{~cm}$, body fat percentage: $13.2 \pm 4.4 \%$ ) volunteered to participate in the study. The sample size was defined a priori by taking jump performance as a reference variable (GPOWER ${ }^{\oplus}$ software) given $\alpha=0.05$, expected power of 0.8 and moderate effect size (0.5). Participants practiced physical exercises (strength training, running and/or sports involving jumps) three to five times a week, for at least one year and had no injuries or pathologies that would preclude maximum effort in the tests. It was required that participants did not perform physical exercises involving the lower limbs $24 \mathrm{~h}$ prior to testing. The experiments were performed in accordance with the ethical standards of the Helsinki Declaration. After an explanation of the procedures, all the participants signed a consent form to participate in the research, which was approved by the ethics committee of the Federal University of Santa Catarina, and guaranteed their rights and anonymity (CAAE= 87443318.9.0000.0121).

\section{Procedures}

At the first visit, participants were familiarized with the jump procedures, and anthropometric measurements were taken to assess the fat percentage (stature, body mass, skinfolds) and kinematic processing (lengths and diameters of the lower limbs). Participants returned on the second day to perform the first fatigue protocol. First, they responded to a perceived exertion scale (Foster et al., 2001) of magnitude 0-10 (0 - no effort; 10 - maximal effort) to monitor rest. Participants performed a warm-up composed of 10 minutes on a cycle ergometer at $90 \mathrm{~W}$. Next, reflective markers (14 $\mathrm{mm}$ ) for kinematic analysis were placed on the right side of the body on the following anatomical landmarks: acromion, major trochanter, anterior superior iliac spine, posterior superior iliac spine, medial thigh, epicondyle lateral femoral, medial leg, lateral malleolus, calcaneus, and proximal phalanx of the hallux. Then, participants performed three maximal countermovement jumps under pre-fatigue conditions (baseline) on two force platforms (AMTI ${ }^{\circledR}$ OR6-7-OP-2000, USA; $2000 \mathrm{~Hz}$ ). They started the jump from a static standing position and were instructed to perform a countermovement followed by a rapid and vigorous extension of the lower limb joints, maintaining their trunk as vertical as possible with their hands on their hips. The participants were then instructed to jump as high as possible, maintaining one foot on each platform prior to take-off and after landing. Immediately following the fatigue protocol, the participants performed three maximal countermovement jumps to represent the post-fatigue condition. All pre- and post-fatigue jumps were filmed $\left(\mathrm{VICON}^{\oplus}, \mathrm{MX}\right.$ systems, UK; $200 \mathrm{~Hz}$ ) for kinematic analysis of movement. At the end of the test session, participants respond to a perceived exertion scale, referring to the total effort applied to perform the fatigue pro- 
tocol. After a minimum of seven days, the subjects returned to the laboratory to perform the fatigue protocol with higher volume. This recovery interval was based on previous studies suggesting that 72 to $96 \mathrm{~h}$ are enough to recover after SSC fatiguing tasks (Byrne \& Eston, 2002; Byrne et al., 2004). In addition, we evaluated muscle soreness before starting the higher-volume protocol using a visual scale (0-10). Soreness values were verified in quadriceps, hamstring, gluteal, and gastrocnemius of $0.0,0.1,0.1$, and 0.1 , respectively, suggesting muscle recovery.

\section{Fatigue protocol}

Two vertical jump protocols were used to induce fatigue: the low-volume protocol was composed of seven sets of 10 continuous jumps; the high-volume was composed of 14 sets of 10 continuous jumps. The interval between sets was one minute for both protocols. Our protocols were based on a jumping protocol previously established for inducing SSC fatigue composed of 10 bouts of 10 countermovement jumps (Byrne et al., 2004; Lazaridis et al., 2018; Twist \& Eston, 2005). In the present study, we slightly reduced the number of bouts to seven for the low-volume protocol and then doubled that number for the high-volume protocol. Our goal was to have a protocol with twice the volume of the other protocol. We verified in a pilot study that both protocols induced fatigue, but with higher levels in the high-volume protocol than in the low-volume protocol. During the jumps, participants were asked to maintain their trunk as vertical as possible, with their hands on their hips, and with a knee angle of $90^{\circ}$ at the end of the descent phase of the jump. The fatigue protocols were performed on a piezoelectric force platform (Kistler ${ }^{\circledast}$ Quattro Jump, 9290 AD, Switzerland; 500 $\mathrm{Hz}$ ). During vertical jumps, it is natural that imbalances occur, and this platform provides a satisfactory space $\left(1 \mathrm{~m}^{2}\right)$ for efficient and safe testing. The participants received verbal encouragement during the protocols to perform all the jumps with the maximum intensity. The fatigue index was calculated as follows in Equation 1 (Dal Pupo et al., 2013), taking into consideration the power output (force multiplied by velocity) during the propulsive phase of the jumps.

$$
\text { Fatigue Index }=\left(\frac{\text { PMEAN }_{4 \mathrm{~J}}-\mathrm{PMEAN}_{\mathrm{END} 4 \mathrm{~J}}}{\mathrm{PMEAN}_{4 \mathrm{~J}}}\right) * 100
$$

Where $\mathrm{PMEAN}_{4 \mathrm{~J}}$ is the average power of the first four jumps, and $\mathrm{PMEANEND}_{4 \mathrm{~J}}$ is the average power of the last four jumps.

\section{Data analysis and dependent variables}

Three-dimensional kinematics of the movement were obtained during the countermovement jumps using a system with eight high-speed integrated cameras $\left(\mathrm{VICON}^{\oplus}, \mathrm{MX}\right.$ systems, UK; $200 \mathrm{~Hz}$ ), which performed the identification of reflective markers through infrared illumination. The system was calibrated and synchronized with the AMTI ${ }^{\circledR}$ force platforms. The three-dimensional coordinates of the reflective markers were filtered using a fourth-order Butterworth low-pass filter with a cut-off frequency of $10 \mathrm{~Hz}$, determined from spectral analysis, following which the reconstruction of the countermovement jumps was done using the plug-ingait model of the Nexus ${ }^{\circledast}$ software (VICON system ${ }^{\circledast}$ ).

The performance in countermovement jump (jump height) was obtained using kinematic analysis; it was con- sidered the highest vertical displacement of the reflective marker placed on the trochanter (Dias et al., 2011). Ground reaction force data obtained during the jumps were filtered using a low-pass, fourth-order Butterworth filter with a cutoff frequency of $10 \mathrm{~Hz}$, determined from spectral analysis. Vertical stiffness was determined by the spring-mass model in the landing phase of the countermovement jump, by the quotient between the peak vertical ground reaction force during the landing phase and the vertical displacement of the centre of mass, determined by the double integration of ground reaction force (Serpell et al., 2012).

From the coordinates of the markers of interest, the segmental angles of the trunk, thigh, and shank (formed between the respective segments and a horizontal plane) were determined for the intralimb coordination analysis. The continuous relative phase (CRP) was calculated to assess the coordination between segments (Dal Pupo et al., 2013; Hamill et al., 1999). The calculation of CRP followed four steps: obtaining the phase diagram, normalizing the phase diagram, obtaining the phase angle, and obtaining the continuous relative phase. For full information on the calculation of CRP, see Hamill et al. (1999). CRP was analysed on the right side of the body for the thigh-trunk and thigh-shank couplings. The CRP was calculated in the descent (i.e., from the moment when the centre of mass velocity became negative until zero) and ascent (i.e., from the moment when the centre of mass velocity became positive until take-off) phase of the countermovement jump. The root mean square was calculated to represent the CRP of each coupling in each phase and used for statistical analysis. CRP values close to $0^{\circ}$ indicate that the segments are more in phase, while values close to $180^{\circ}$ indicate that the segments are more out of phase. An algorithm implemented in MatLab ${ }^{\circledast}$ software (The MathWorks, Inc ${ }^{\oplus}$, USA) was used to obtain all kinematic data.

\section{Statistical analysis}

Data are presented as mean \pm standard deviation. A mixed-model analysis of variance (ANOVA) was used to compare the variables between conditions (low and high volume) and within the times (before vs. after). The sphericity of the data was tested (Mauchly's Test) and corrected (Green-house Geiser) when necessary. The significant P-value adopted was $\mathrm{p} \leq 0.05$. Statistical procedures were performed using the Statistical Package for Social Sciences software (v. 17.0, IBM Co., USA).

\section{Results}

The fatigue index was greater in the high-volume protocol $(27.0 \pm 11.8 \%)$ than in the low-volume protocol $(15.8 \pm 7.0 \%)(\mathrm{ES}=1.51)$. Consequently, the rate of the perceived exertion scale of the low-volume protocol (4.8 \pm 1.5$)$ was inferior $(E S=1.69)$ to that of the high-volume protocol (7.5 \pm 1.4$)$.

Jump height showed the interaction between time and protocol $(\mathrm{F}=5.80 ; \mathrm{p}=0.02)$. Post-hoc analysis revealed a decrease in jump height (Figure 1a) after the high-volume protocol $(\mathrm{p}<0.01)$, while no change was observed after the low-volume protocol $(p=0.3)$. No interaction between time and protocols was obtained for vertical stiffness $(\mathrm{F}=0.46$; $\mathrm{p}=0.50)$. However, an effect was visualized in time $(\mathrm{F}=4.09$; $\mathrm{p}=0.05$ ), indicating that vertical stiffness decreased after both protocols (Figure $1 \mathrm{~b}$ ). 
a)

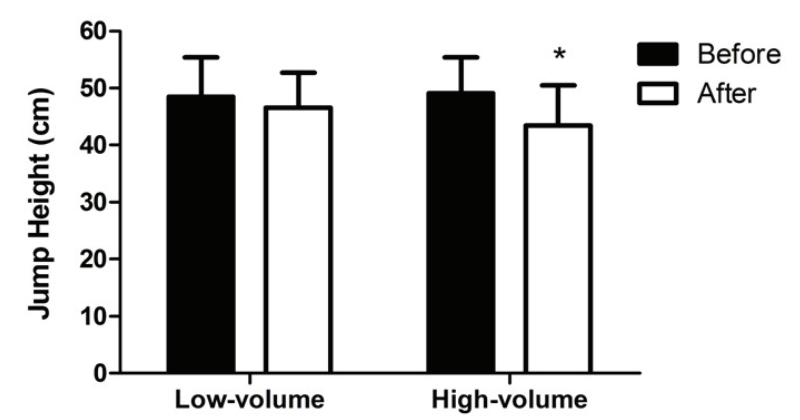

b)

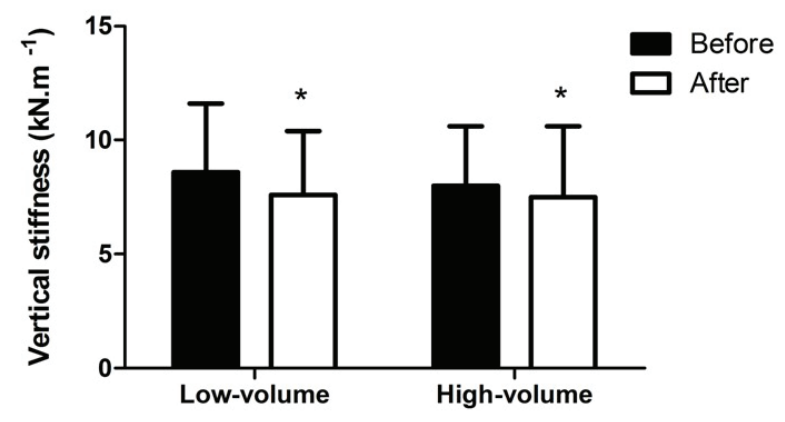

Figure 1. Jump height (panel A) and vertical stiffness (panel B) before and after the low-volume and high-volume protocols. * indicate difference from before-condition

For the intralimb coordination, there was no interaction time vs protocol for thigh-trunk coupling in the descent $(\mathrm{F}=0.55 ; \mathrm{p}=0.46)$ or ascent phase $(\mathrm{F}=0.11 ; \mathrm{p}=0.73)$, nor time effect (descent phase: $\mathrm{F}=0.16, \mathrm{p}=0.69$; ascent phase: $\mathrm{F}=1.07$, $\mathrm{p}=0.31$ ) or protocol (descent phase: $\mathrm{F}=0.06, \mathrm{p}=0.81$; ascent phase: $\mathrm{F}=0.01, \mathrm{p}=0.95)$. Similarly, thigh-shank coupling did not present time $\mathrm{x}$ protocol interaction for descent $(\mathrm{F}=1.52$; $\mathrm{p}=0.23)$ and ascent phase $(\mathrm{F}=0.18 ; \mathrm{p}=0.67)$. However, it was verified time effect $(\mathrm{F}=4.43, \mathrm{p}=0.04)$ of $\mathrm{CRP}$ thigh-shank during the ascent phase of movement, indicating an increase when comparing before vs. after both protocols. The results from the intralimb coordination can be seen in Figure 2.
Low-volume Protocol

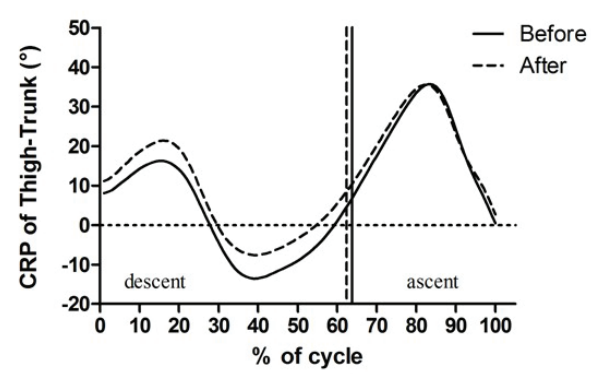

Low-volume Protocol

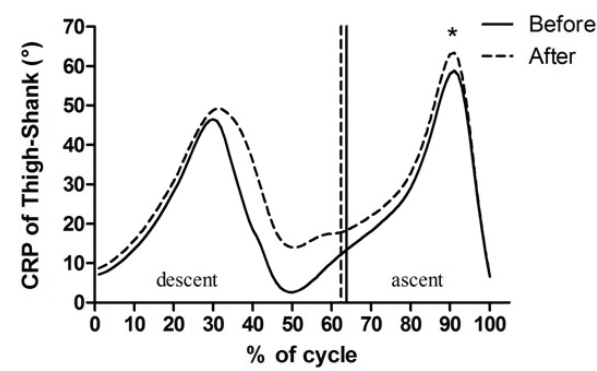

High-volume Protocol

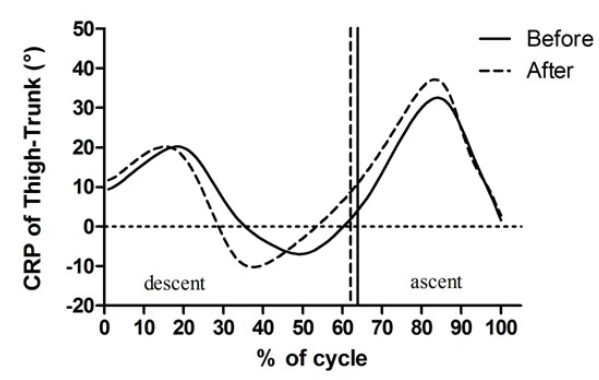

High-volume Protocol

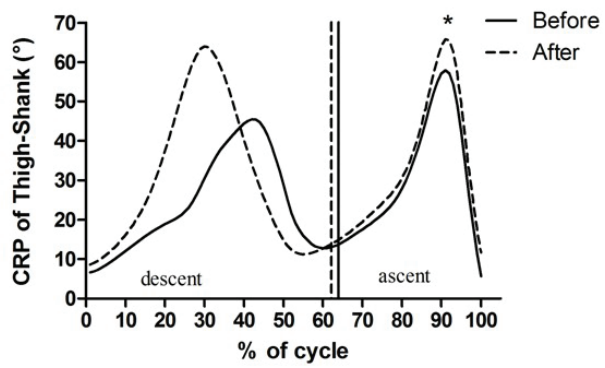

Figure 2. Intralimb coordination (CRP) of thigh-trunk and thigh-leg couplings, for both protocols, during ascent and descent phases of countermovement jump. CRP - continuous relative phase, ${ }^{*}$ indicate difference form before-condition. The vertical lines indicate the transition from phase one for phase two of movement.

\section{Discussion}

This study aimed to investigate the acute effects of different levels of muscular fatigue on vertical jump performance, vertical stiffness, and intralimb coordination. It was observed that by doubling the volume of the fatigue protocol while maintaining the same effort (i.e., maximum jumps), the fatigue level increased from $16.0 \%$ to $27.0 \%$. The main findings indicate that jump performance was affected only by higher fatigue indexes, while vertical stiffness and intralimb coordination were similarly affected irrespective of the levels of fatigue. Thus, the main hypothesis of this study was accepted only for jump performance.
It is known that the high mechanical stress produced by SSC movements induces acute fatigue, which is commonly related to the accumulation of metabolites, energy depletion, and changes in calcium release/reabsorption (Sahlin, 1992; Williams \& Klug, 1995). This results in an impairment of muscle function (Nicol et al., 2006), which consequently may explain the decrease in jump height and vertical stiffness immediately following the fatiguing protocols. However, changes in jump performance seem to occur only when greater mechanical stress is imposed, as seen in the high-volume protocol. Nicol et al. (2006) determined that sometimes exercises with low fatigue indexes reveal facilitation of performance, whereas 
only tests involving high-intensity maximal or near-maximal tests reveal performance deterioration more clearly.

For vertical stiffness, it was observed that SSC fatigue induced a similar decrease immediately following both protocols, suggesting that acute changes in stiffness are not related to the fatigue levels. Most studies with a similar design involving vertical jumps have reported a decrease in vertical and lower limb stiffness after fatigue (Dal Pupo et al., 2013; Horita et al., 1996; Kuitunen et al., 2002; Kuitunen et al., 2007; Lazaridis et al., 2018), although they did not assess or discuss the influence of different levels of fatigue. The decrease in stiffness has been attributed to factors such as a reduction in pre-activation, and alterations in stretching reflex of the triceps sural and knee extensor muscles. These changes may affect the braking capacity of the movement, allowing for greater excursion of movement and consequently decreasing stiffness (Dal Pupo et al., 2013; Kuitunen et al., 2002; Kuitunen et al., 2007; Lazaridis et al., 2018). Lazaridis et al. (2018), Hughes and Watkins (2008), and Dal Pupo et al. (2013) suggest that alterations can occur as a protective mechanism against injury.

The intralimb coordination in the thigh-trunk (in both phases of movement) and thigh-shank (descent phase) couplings did not change after any of the fatigue protocols. The absence of changes in coordination at fatigue conditions has been attributed to the presence of a pre-programmed "common drive", which is difficult to change and that guides the muscle activation and, consequently, the coordination, maintaining the movement pattern through fixed neural commands, even under fatigue conditions (Rodacki et al., 2001). However, we verified that thigh-shank coupling CRP values increased after both protocols, indicating that the movement between the segments became more out of phase after fatigue. Although the thigh-trunk coupling generates important angular moments during a vertical jump (Gheller et al., 2015), the changes observed only in thigh-shank coupling suggest that the muscles around the knee joint are more affected by fatigue. The modulation of coordinative patterns after a fatiguing activity has been suggested as a compensation of the motor control system in an attempt to maintain the performance of the task despite decreased force production (Dal Pupo et al., 2013; Madigan \& Pidcoe, 2003). Dal Pupo et al. (2013) suggest that the presence of fatigue or even the modulation of stiffness may influence muscle spindles (and consequently on proprioceptors), which would send afferent feedback signals to the central nervous system to rearrange the coordination pattern of the segments. Moreover, changes in coordination are also speculated as a protective mechanism against injuries, aiming to increase the stability of the limbs or even decrease the ground reaction forces experienced (Hughes \& Watkins, 2008; Madigan \& Pidcoe, 2003). In addition, the changes to the CPR thigh-shank coupling occurred during the ascent phase of the countermovement jump, which is considered the most determinant for jump performance development (Dal Pupo et al., 2012; Kirby et al., 2011), reinforcing that changes in coordination may have occurred to maintain the vertical jump performance.

The results showed that the fatigue effect was task-dependent (different volumes) for jump performance, in which longer exercise caused more fatigue and consequently induced greater impairment in muscle function (Enoka \& Stuart, 1992; Nicol et al., 2006); however, this "linear" response was not evident for motor control parameters, such as vertical stiffness and intralimb coordination. Both vertical stiffness and CRP of thigh-shank coupling had similar changes after the fatigue protocols (i.e., different volumes induced the same changes). According to our results, it seems to exist a minimum fatigue threshold at which these changes occur, provoking the same alterations independent of the fatigue index. Nicol et al. (2006) suggest that in maximal exercises a neural attempt of protection of the fatigued muscle, which does not occur in less fatiguing exercises, may exist. Thus, it can be speculated that the similar effects visualized after both protocols are related to this protective mechanism acting during the high-volume protocol (maximal exercises) but not during the low-volume protocol.

The present study has limitations. The evaluations post-fatigue were conducted with the shortest possible interval after the application of the protocol; however, the time between the completion of the protocol and the evaluation was on average 1 minute and 20 seconds. This may have allowed some level of recovery, influencing the results. In addition, due to methodological limitations, it was not possible to compose a sample of athletes. Thus, although the participants in the present study are physically active, it is difficult to generalize the results to an athlete population since the neuromuscular responses to fatigue could be different due to the level of training. Further studies with a similar theme, involving a population of athletes, are encouraged.

In conclusion, only the high-volume fatigue protocol, which induced a high rate of perceived exertion and fatigue index, was able to decrease the jump performance. In contrast, the decrease in vertical stiffness and in the intralimb coordination of thigh-shank coupling occurred after both protocols, suggesting that changes is these parameters related to movement control are irrespective of the fatigue levels. From a practical point of view, the results of the present study may be important throughout a competition or match when the fatigue is progressive. If we consider, for example, applying our results to a football match, the player will only lose performance significantly after increased levels of accumulated fatigue, probably at the end of the match. However, greater fatigue did not necessarily lead to more commitment in the coordination pattern and vertical stiffness. This suggests that already with low levels of fatigue, changes in stiffness and coordination may occur, probably to keep the control of movement to maintain performance and as a protective neural mechanism against injuries that may occur upon high impact with the ground.

\section{Acknowledgements}

We would like to thank the Coordenação de Aperfeiçoamento de Pessoal de Nível Superior (CAPES), for the scholarship provided to the first author while conducting this project.

\section{References}

Byrne, C., \& Eston, R. (2002). The effect of exerciseinduced muscle damage on isometric and dynamic knee extensor strength and vertical jump performance. Journal of Sports Science, 20(5), 417-425. https://doi. org/10.1080/026404102317366672

Byrne, C., Twist, C., \& Eston, R. (2004). Neuromuscular Function After Exercise-Induced Muscle Damage Theoretical and Applied Implications. Sports Medicine, 34(1), 49-69. https://doi.org/10.2165/00007256200434010-00005

Dal Pupo, J., Ache Dias, J., Gheller, R. G., Detanico, D., \& 
Santos, S. G. (2013). Stiffness, intralimb coordination, and joint modulation during a continuous vertical jump test. Sports Biomechanics, 12(3), 259-271. https://doi.org/10.10 80/14763141.2013.769619.

Dal Pupo, J., Detanico, D., \& Dos Santos, S. G. (2012). Kinetic parameters as determinants of vertical jump performance. Revista Brasileira de Cineantropometria e Desempenho Humano, 14(1), 41-51. https://doi.org/10.5007/19800037.2012v14n1p41

Dias, J. A., Dal Pupo, J., Reis, D. C., Borges, L., Santos, S. G., Moro, A. R., \& Borges, N. G. Jr. (2011). Validity of two methods for estimation of vertical jump height. Journal of Strength and Conditioning Research, 25(7), 2034-2039. https://doi.org/10.1519/JSC.0b013e3181e73f6e

Enoka, R.M., \& Stuart, D.G. (1992). Neurobiology of muscle fatigue. Journal of Applied Physiology, 72(5), 1631-1648. https://doi.org/10.1152/jappl.1992.72.5.1631

Foster, C., Florhaug, J. A., Franklin, J., Gottschall, J. L., Hrovatin, L. A., Parker, S. B., Doleshal, P., \& Dodge, C. (2001). A new approach to monitoring exercise training. Journal of Strength and Conditioning Research, 15(1), 109115. https://doi.org/10.1519/00124278-200102000-00019

Gheller, R. G., Dal Pupo, J., Ache-Dias, J., Detanico, D., Padulo, J., \& dos Santos, S. G. (2015). Effect of different knee starting angles on intersegmental coordination and performance in vertical jumps. Human Movement Science, 42, 71-80. https://doi.org/10.1016/j.humov.2015.04.010

Hamill, J., Van Emmerik, R. E. A., Heiderscheit, B. C., \& Li, L. (1999). A dynamical systems approach to lower extremity running injuries. Clinical Biomechanics, 14(5), 297-308. https://doi.org/10.1016/S0268-0033(98)90092-4

Horita, T., Komi, P. V., Nicol, C., \& Kyröläinen, H. (1996). Stretch shortening cycle fatigue: interactions among joint stiffness, reflex, and muscle mechanical performance in the drop jump. European Journal of Applied Physiology, 73(5), 393-403. https://doi.org/10.1007/BF00334415

Hughes, G., \& Watkins, J. (2008). Lower limb coordination and stiffness during landing from volleyball block jumps. Research in Sports Medicine, 16(2), 138-154. https://doi. org/10.1080/15438620802103999

James, R. C., Dufek, J. S., \& Bates, B. T. (2006). Effects of Stretch Shortening Cycle Exercise Fatigue on Stress Fracture Injury Risk During Landing. Research Quarterly for Exercise and Sport, 77(1), 1-13. https://doi.org/10.1080 /02701367.2006.10599346

Kirby, T. J., McBride, J. M., Haines, T. L., \& Dayne, A. M. (2011). Relative net vertical impulse determines jumping performance. Journal of Applied Biomechanics, 27(3), 2072014. https://doi.org/10.1123/jab.27.3.207

Komi, P. V. (2000). Stretch-shortening cycle: a powerful model to study normal and fatigued muscle. Journal of Biomechanics, 33(10), 1197-1206. https://doi.org/10.1016/ S0021-9290(00)00064-6

Kuitunen, S., Avela, J., Kyröläinen, H., Nicol, C., \& Komi, P. V. (2002). Acute and prolonged reduction in joint stiffness in humans after exhausting stretch-shortening cycle exercise. European Journal of Applied Physiology, 88(1-2), 107-116. https://doi.org/10.1007/s00421-002-0669-2.

Kuitunen, S., Kyröläinen, H., Avela, J., \& Komi, P. V. (2007). Leg stiffness modulation during exhaustive stretch-shortening cycle exercise. Scandinavian Journal of Medicine and Science in Sports, 17(1), 67-75. https://doi.org/10.1111/ j.1600-0838.2005.00506.x

Lazaridis, S., Patikas, D. A., Bassa, E., Tsatalas, T., Hatzikotoulas, K., Ftikas, C., \& Kotzamanidis, C. (2017). The acute effects of an intense stretch-shortening cycle fatigue protocol on the neuromechanical parameters of lower limbs in men and prepubescent boys. Journal of Sports Sciences, 8(2), 1-9. https://doi.org/10.1080/02640414.2017.1287932

Madigan, M. L., \& Pidcoe, P. E. (2003). Changes in landing biomechanics during a fatiguing landing activity. Journal of Electromyography and Kinesiology, 13(5), 491-498. https://doi.org/10.1016/S1050-6411(03)00037-3.

McMahon, J. J., Comfort, P., \& Pearson, S. (2012). Lower limb stiffness: Effect on performance and training considerations. Strength \& Conditioning Journal, 34(6), 94-101. https://doi.org/10.1519/SSC.0b013e3182781b4e

Mudie, K. L., Gupta, A., Green, S., \& Clothier, P. J. (2016). Adaptation of lower limb movement patterns when maintaining performance in the presence of muscle fatigue. Human Movement Science, 48, 28-36. https://doi. org/10.1016/j.humov.2016.04.003

Nicol, C., Avela, J., \& Komi, P. (2006). The StretchShortening Cycle A Model to Study Naturally Occurring Neuromuscular Fatigue. Sports Medicine, 36(11), 977-999. https://doi.org/10.2165/00007256-200636110-00004

Padua, D. A., Arnold, A. L., Perrin, D. H., Gansneder, B. M., Carcia, C. R., \& Granata, K. P. (2006). Fatigue, vertical leg stiffness and stiffness control strategies in males and females. Journal of Athletic Training, 41(3), 294-304.

Rodacki, A. L. F., Fowler, N. E., \& Bennett, S. J. (2001). Multisegment coordination: Fatigue effects. Medicine and Science in Sports and Exercise, 33(1), 1157- 1167. https:// doi.org/10.1097/00005768-200107000-00013

Rodacki, A. L. F., Fowler, N. E., \& Bennett, S. J. (2002). Vertical jump coordination: Fatigue effects. Medicine and Science in Sports and Exercise, 34(7), 105-116. https:/doi. org/10.1097/00005768-200201000-00017

Sahlin, K. (1992). Metabolic factors in fatigue. Sports Medicine, 13(2), 99-107. https://doi.org/10.2165/00007256199213020-00005

Serpell, B. G., Ball, N. B., Scarvell, J. M., \& Smith, P. N. (2012). A review of models of vertical, leg and knee stiffness in adults for running, jumping or hopping tasks. Journal of Sports Sciences, 30(13), 1347-1363. https://doi.org/10.108 $0 / 02640414.2012 .710755$

Twist, C., \& Eston, R. (2005). The effects of exercise-induced muscle damage on maximal intensity intermittent exercise performance. European Journal of Applied Physiology, 94(5-6), 652-658. https://doi.org/10.1007/s00421-0051357-9

Williams, J. H., \& Klug, G. (1995). Calcium exchange hypotesis of skeletal muscle fatigue: a brief review. Muscle and Nerve, 18(4), 421-434. https://doi.org/10.1002/mus.880180409

Zhang, X., Xia, R., Dai, B., Sun, X., \& Fu, W. (2018). Effects of exercise-induced fatigue on lower extremity joint mechanics, stiffness, and energy absorption during landings. Journal of Sports Science \& Medicine, 17(4), 640-649. 